\title{
The Role of Genetics in Nicotine Dependence: Mapping the Pathways from Genome to Syndrome
}

\author{
James MacKillop, $\mathrm{PhD}^{1}$, Ezemenari Obasi, $\mathrm{PhD}^{2}$, Michael T. Amlung, MS ${ }^{3}$, John E. \\ McGeary, $\mathrm{PhD}^{4}$, and Valerie S. Knopik, $\mathrm{PhD}^{5}$ \\ James MacKillop: jmackill@uga.edu; Ezemenari Obasi: obasi@uga.edu; Michael T. Amlung: mamlung@uga.edu; John E. \\ McGeary: John_McGeary@Brown.edu; Valerie S. Knopik: Valerie_Knopik@Brown.edu \\ ${ }^{1}$ Department of Psychology, University of Georgia; Center for Alcohol and Addiction Studies, \\ Brown University \\ 2 Department of Counseling and Human Development Services, University of Georgia \\ ${ }^{3}$ Department of Psychology, University of Georgia \\ ${ }^{4}$ Substance Abuse Treatment Program; Providence Veterans Administration Medical Center; \\ Division of Behavioral Genetics, Department of Psychiatry, Rhode Island Hospital; Department of \\ Psychiatry and Human Behavior, Brown University \\ ${ }^{5}$ Division of Behavioral Genetics, Department of Psychiatry, Rhode Island Hospital; Department \\ of Psychiatry and Human Behavior, Brown University
}

\begin{abstract}
Nicotine dependence continues to be a major public health problem worldwide and there is unequivocal evidence that genetics play a substantial role in its etiology. This review provides an overview of the evidence for genetic influences and recent advances in the field. Traditional quantitative genetics studies have revealed nicotine dependence is heritable and molecular genetics studies are providing increasing evidence that the genes responsible for nicotine's pharmacokinetics and pharmacodynamics are particularly important. Despite considerable progress, a number of significant complexities and challenges remain. These include determining the specificity of genetic influences and clarifying the role of interactive contributions. One promising strategy for addressing these issues is an intermediate phenotype approach that attempts to identify the intervening proximal mechanisms that confer differential genetic risk. Understanding these mechanisms may permit more precision in understanding genetic influences and may also identify novel targets for intervention or prevention.
\end{abstract}

\section{Keywords}

Nicotine Dependence; Genetics; Smoking; Tobacco; Intermediate Phenotype; Endophenotype

\section{Introduction}

Cigarette smoking is the single largest cause of preventable morbidity and mortality in the United States and the world [1]. Smoking is associated with increased risk for lung cancer, heart disease, and chronic obstructive pulmonary disease and estimated to result in

Corresponding Author: James MacKillop, PhD, jmackill@uga.edu; tel. 706.542.3033.

Disclosure

No potential conflicts of interest relevant to this article were reported. 
approximately 450,000 deaths annually in the United States alone [2]. Reducing the prevalence of smoking via treatment and prevention is a major international public health priority. For the majority of smokers, tobacco use is specifically motivated by nicotine dependence, as nicotine is the primary compound responsible for tobacco reinforcement [3]. As such, understanding the causes of nicotine dependence is essential for decreasing the prevalence of smoking. Unequivocal evidence has demonstrated genetic factors play a substantial role in nicotine dependence, and this review provides an overview of the research in this area. Given the extensive body of work, the goal is not to be exhaustive but rather to synthesize the overall patterns of findings in humans. We use a top-down approach, first reviewing evidence of the heritability of nicotine dependence and then focusing on the specific sources of genetic influences. Finally, current challenges and the application of an intermediate phenotype approach as a promising strategy for progress are reviewed.

\section{Quantitative Genetic Influences}

Numerous quantitative genetic studies have demonstrated a substantial genetic contribution to many aspects of smoking behavior and nicotine dependence. The quantitative approach incorporates family, adoption, and twin designs that capitalize on the comparison of relatives that differ in levels of genetic relatedness. For example, early twin studies simply compared concordance of smoking behavior in monozygotic (identical) and dizygotic (fraternal) twin pairs. More recent approaches to twin analyses incorporate statistical models that permit parameter estimation of genetic and environmental contributions to individual differences in the predisposition to smoking and related behaviors (for a review, see Rose et al. [4••]). This genetic contribution, or heritability, is population and time specific and can be defined as the proportion of the total phenotypic variance that is due to genetic effects. Heritability can reflect not only sampling variability, but also true variation due to multiple factors affecting smoking prevalence and the likelihood that a genetic predisposition can become manifest. Such factors might include birth cohort, religiosity, family structure and status, parental smoking history, socioeconomic conditions, and availability of tobacco. Importantly, the heritability of the clinical phenotype of nicotine dependence remains consistent ( $50 \%)$ across many studies, assessment measures, and cultures [4••], as does the heritability of volumetric tobacco consumption (eg, cigarettes per day), which is also estimated at about $50 \%[4 \bullet \bullet]$.

Another strategy for examining the heritability of smoking behavior is to parse its chronology, examining both initiation and persistence. In a meta-analysis of 16 such studies, $\mathrm{Li}$ et al. [5] found both phenotypes were substantially heritable but also observed potentially meaningful sex differences. Across studies, the heritability of smoking initiation was 39\% for men and 55\% for women, whereas the heritability of smoking persistence was 59\% for men and $46 \%$ for women. Thus, genetic influences appear to play a larger role for women in terms of starting to smoke and are more important for men when considering smoking progression. However, a more recent review that included several new studies with larger samples supported the evidence of substantial genetic influences on both smoking initiation and persistence but not sex differences [4••]. Moreover, there is little evidence of common genetic effects that influence both initiation and persistence [6], suggesting that there are genetic processes contributing to experimentation and initiation that are distinct from those influencing maintenance of a longstanding habit. There is also evidence of significant differences in heritability based on age. Specifically, genetic effects tend to be stronger as age increases [7], suggesting that environmental factors contribute early in life and genetic factors predominate later in life.

In sum, quantitative genetic studies have made, and continue to make, major contributions to our knowledge of the genetic epidemiology of smoking behavior and nicotine dependence. 
There is a substantial genetic contribution to these behaviors that consistently accounts for roughly half of the phenotypic variance; however, there do appear to be meaningful differences in the genetic contribution across the lifespan. Further, whereas genetic factors significantly influence both smoking initiation and persistence individually, there is no evidence of a significant genetic contribution to the covariation between these two phenotypes.

\section{Molecular Genetic Influences}

Beyond evidence of latent additive genetic effects, which provides no information about what specific genes are involved, there is an increasing understanding of the individual molecular variants that confer risk for nicotine dependence. The most consistent evidence suggests that the genes responsible are those associated with differences in nicotine's pharmacokinetics (ie, metabolic capacity) and pharmacodynamics (ie, central nervous system neurotransmitter functionality). A model of these influences is presented in Figure 1, including the primary metabolic pathways, neurotransmitter systems, and a number of illustrative candidate genes.

\section{Genetic variation influencing nicotine pharmacokinetics}

Nicotine's pharmacokinetics are relatively well understood. Approximately $75 \%$ of nicotine is metabolized into cotinine, which is itself partially metabolized into trans-3'hydroxycotinine. In both cases, these conversions are catalyzed by liver cytochrome P450 enzymes (CYPs), primarily the CYP2A6 enzyme. The efficiency of this metabolic pathway varies considerably across individuals and is substantially influenced by genetic variation (for a review, see Mwenifumbo and Tyndale [8]). Twin studies indicate approximately 60\% of the variability in nicotine metabolism is attributable to genetic factors [9]. The major genetic contributor to variation in this pathway is the enzyme's eponymous gene, CYP2A6, which is part of a cluster of six CYP genes and is highly polymorphic. The most common ("wild-type") allele is designated $C Y P 2 A 6 * 1$, and an array of other polymorphisms have been identified, including deletions, duplications, insertions, and single nucleotide polymorphisms (SNPs). The functional effects of many of these polymorphisms are unknown but a number of them substantially reduce enzymatic metabolism of nicotine and others increase nicotine metabolism. In terms of magnitude, CYP2A6 has major effects on metabolic capacity; individuals who are homozygous for the gene deletion exhibit approximately $400 \%$ greater levels of nicotine, and possession of one decreased-activity allele has been found to be associated with approximately $50 \%$ lower enzymatic activity [8]. These differences are striking, but there is also substantial variation among wild-type carriers [10], suggesting the importance of other sources of variation, genetic or otherwise.

Possession of a decreased-activity allele has been found to be generally protective, with individuals being less likely to smoke and, among smokers, associations with lower cigarette consumption and greater probability of successful smoking cessation [11, 12]. In addition, in a laboratory study on smoking topography, individuals with decreased-activity alleles exhibited significantly lower puff volume and total puff volume, although not number of puffs [13]. However, a number of studies have not identified systematic differences based on CYP2A6 genotype, creating an overall pattern of mixed findings (for a meta-analysis, see Munafò et al. [14]).

A second candidate in this pathway is CYP2B6, which also metabolizes nicotine in the liver, albeit less efficiently than CYP2A6 [8]. The role of CYP2B6 has not been extensively investigated, and the published studies on its relevance are mixed $[15,16]$. Of note, a particularly interesting recent finding is that $C Y P 2 A 6$ and $C Y P 2 B 6$ genotype interact in relation to nicotine metabolism. A two-locus CYP2B6 haplotype has been associated with 
faster nicotine metabolism and was more pronounced for individuals with decreased activity CYP2A6 genotypes [17]. This suggests CYP2B6 may be particularly important for understanding variability in individuals with decreased CYP2A6 activity.

Although considerably less studied, there are other aspects of nicotine's pharmacokinetics that may also be meaningfully affected by genetics. A small percentage of nicotine is metabolized into nicotine $N^{\prime}$-oxide by flavin-containing monooxygenase 3 (FMO3) and excreted in urine. Moreover, individuals who are homozygous for the CYP2A6 gene deletion excrete about four times more nicotine this way [18], indicating an alternative metabolic pathway. Considerable inter-individual variability is evident in this pathway and a large number of polymorphisms have been identified in the FMO3 gene, but no studies to date have investigated $F M O 3$ genotype in relation to nicotine dependence. Finally, another process in nicotine metabolism is the glucuronidation of nicotine, cotinine, and trans-3'hydroxycotinine to facilitate urinary excretion. Glucuronidation takes place via uridinediphosphate glucuronosyltransferase enzymes (UGTs) and for which very high levels of variability in activity are evident [8]. This is a second alternative metabolic route for individuals who are homozygous for CYP2A6 deletions. The specific enzymes are UGT2B10 (nicotine and cotinine) and UGT2B7 (trans-3'-hydroxycotinine), and numerous polymorphisms in the $U G T 2 B$ subfamily of genes have been identified. Moreover, the $U G T 2 B 10 * 2$ allele has been associated with reduced nicotine and cotinine glucuronidation [19], making it a promising candidate.

\section{Genetic variation influencing nicotine pharmacodynamics}

The molecular pharmacology of nicotine's psychoactive effects is via direct and indirect effects of nicotinic cholinergic receptor (nAChR) excitation (for a review, see Benowitz [20]). Nicotine directly enhances cognitive functioning, such as attention, learning, and memory, via $\mathrm{nAChR}$ stimulation. In addition, $\mathrm{nAChR}$ excitation provokes burst firing in subcortical dopamine-secreting neurons that are part of the mesocorticolimbic DA pathway. The dynamic increase in dopamine is believed to be responsible for the reinforcing effects of nicotine and its addiction potential. Indeed, all addictive drugs commonly activate this dopaminergic circuitry [21]. In addition to nAChR-mediated increases in dopamine, nicotine also augments dopamine release via glutamatergic, GABAergic, and opioidergic mechanisms. Over time, chronic nicotine exposure leads to neuroadaptive receptor upregulation and greater nicotine-elicited dopamine release, further strengthening dependence [20].

Genetic variation that contributes to functional differences within these circuits is both a logically plausible source of altered risk for nicotine dependence and one for which there is also considerable empirical evidence. The most robust findings to date have implicated variation in the genes that are responsible for nAChRs. Pharmacologically, nAChRs are composed of varying combinations of five $\alpha$ and $\beta$ subunits. Functionally, nAChR subtypes vary widely, but the $\alpha 4 \beta 2 \mathrm{nAChRs}$ have a particularly high affinity for nicotine. Indeed, there is evidence that $\alpha 4$ subunit nAChRs are sufficient for nicotine reinforcement, tolerance, and sensitization [22], and functional neuroimaging has revealed that experiential satiation from smoking closely scales to $\alpha 4 \beta 2$ occupancy [23]. Moreover, a number of studies have found that variation in CHRNA4, the $\alpha 4$ subunit gene, influences nicotine dependence [24••]. In addition, potentially important genetic variation in cholinergic neurotransmission has been identified in a cluster of genes responsible for the $\alpha 5, \alpha 3$, and $\beta 4$ nAChR subunits [25, 26]. One locus (rs16969968) in the $\alpha 5$ subunit gene, CHRNA5, appears to be particularly important, with a recent meta-analysis revealing a highly significant association with smoking level in over 38,000 smokers $\left(P<10^{-35}\right)[27 \bullet \bullet]$. 
Beyond cholinergic genes, a second logical target is genetic variation in the other neurotransmitter systems associated with nicotine's pharmacodynamics, such as the dopamine and endogenous opioid systems. In the first case, dopamine is enzymatically broken down by dopamine $\beta$ hydroxylase and catechol-O-methyltransferase, which are encoded by the $D B H$ and $C O M T$ genes, respectively. A number of studies have found associations between polymorphisms in $D B H$ and COMT and nicotine dependence or other smoking-related phenotypes [28, 29]. In addition, polymorphisms in or linked to the genes encoding the dopamine receptor subtypes have been associated with smoking status and motivation for cigarettes [30-33], as well as smoking cessation treatment outcome [34]. With regard to opioid neurotransmission, although there have been fewer studies, there is evidence for associations between polymorphisms in the $\mu$ opioid receptor gene, OPRM1, and several smoking phenotypes, including initiation of smoking [35], the reinforcing value of smoking [36], and treatment outcome [37].

The preceding genes and polymorphisms are by no means the only ones associated with nicotine dependence, but they reflect the findings for which there is the greatest convergence of evidence. Many additional loci have also been identified, especially in recent studies using high-throughput genotyping technologies, such as genome-wide association studies. These studies can assess millions of polymorphisms at once and have both supported the relevance of loci in the preceding systems and implicated a number of heretofore unidentified loci [38]. It is also important to note, however, that there have been many failures to replicate results, and there have even been significant findings in opposite directions, leading to an often conflicting and inconsistent literature [14]. Moreover, where there are relatively reliable associations, the magnitudes of effects tend to be small. As such, there is little evidence of a "nicotine-dependence gene" or even a small number of highimpact variants that largely explain its high heritability. Instead, risk for tobacco dependence appears to be conferred by a large number of genetic polymorphisms of relatively small magnitude effects, and even this interpretation cannot be conclusive in light of a number of persistent challenges.

\section{Complexities and Challenges}

There are a numerous complexities to understanding the genetics of nicotine dependence, but we focus on two of the most significant issues, namely, ambiguity about the specificity of genetic influences and uncertainty about the extent to which interactive relationships play a role. These issues have the potential to be the largest causes of inconsistency in the field and unraveling them will be necessary to make a full account of genetic influences.

In the first case, specificity refers to the extent to which genetic influences directly alter risk for nicotine dependence but not other characteristics or conditions. A highly specific risk variant would only increase or decrease the probability of developing nicotine dependence, whereas a relevant but nonspecific variant might be tangentially related to nicotine dependence by altering risk for other variables that are probabilistically relevant to tobacco use. Characterizing specificity is not just important for validly understanding the role of a given variant, but it is methodologically essential because nonspecific variants could introduce "third variable" confounds (ie, unmeasured variables that are causally responsible for associations spuriously observed between two other variables). From a statistical standpoint, this is a problem because the significance of an association study would depend on the frequency of the unmeasured variable. This would consequently be expected to generate mixed findings based on samples with different latent characteristics on the unmeasured variable and to underestimate the magnitude of the influence by inadvertently combining individuals for whom it is relevant and those for whom it is not. Moreover, it is also possible that a locus be associated with an entirely unrelated variable. A prototypic 
example of this is population stratification [39], which is a confounding of the phenotype of interest with racial/ethnic status and, in turn, spurious associations with polymorphisms that also systematically vary by race or ethnicity.

Unfortunately, it is often an implicit assumption that there is a relatively high level of specificity between genetic factors and nicotine dependence, but there is a strong empirical basis for a large portion of genetic heritability to come from indirect influences. First, from a purely epidemiologic standpoint, nicotine dependence is comorbid with a number of psychiatric conditions, such as depression, schizophrenia, and substance use disorders [40], which are also genetically influenced and would be expected to indirectly affect motivation to smoke. Second, twin studies have identified large nonspecific genetic effects on substance use disorders and conduct disorder/antisociality [41] and shared genetic effects on licit substance use (tobacco and alcohol) [42]. Third, as noted above, all addictive drugs activate the mesocorticolimbic dopamine pathway [21], and nAChRs are both highly expressed in this pathway and serve in a modulatory capacity [20], suggesting that genetically mediated sensitivity within this system may not be specific to risk for nicotine dependence. Finally, there are a number of parallel behavioral characteristics across types of substance dependence, such as impulsivity and reactivity to drug stimuli, and there is evidence these are influenced by common genetic variants $[32,43]$. Thus, indirect genetic pathways may contribute to contradictory findings across studies and ambiguity about the level of specificity of genetic influences represents both a significant gap in knowledge and a serious methodologic challenge.

The second major challenge pertains to understanding the role of interactive relationships in genetic risk for tobacco dependence. The majority of studies primarily examine independent associations between one or a small number of polymorphisms and a smoking-related phenotype. However, genetic influences may be conferred as a result of a number of different interactive relationships. At the simplest level, genetic influences may not pertain to genotypes, but rather haplotypes (ie, common combinations of proximal variants that are inherited together) [44]. In such cases, if the other variants comprising the haplotype are not assessed, a latent unmeasured source of error is again introduced. Likewise, there may also be gene-gene interactions (ie, epistasis) between loci that are independently related to nicotine dependence [17]. Possession of multiple alleles that confer greater risk by small amounts may multiplicatively increase the probability of nicotine dependence (ie, based on non-additive interactions, a person's total genetic risk may be greater than sum of its parts). Beyond the level of the genome, more complex interactions include those between genetic variables and nongenetic (environmental) variables, such as developmental events (eg, age of initiation [45]) and the influences of acute experiential states, such as stress, craving, withdrawal, and negative affect [46]. A good example of these multiple sources of complexity is evident in a recent study of the CHRNA5-A3-B4 gene cluster in relation to nicotine dependence and other smoking-related phenotypes [26]. In this case, a haplotypebased analysis was more sensitive than examining SNPs individually, revealing both risk and protective combinations, and the associations with nicotine dependence were only significant in individuals who began smoking at an early age.

A further complication is that there is increasing evidence that epigenetic processes contribute to addiction [47], and nicotine dependence in particular [48]. Epigenetic mechanisms are cellular level modifications to genetic material based on environmental exposures (eg, diet, chronic stress, drug exposure) that alter subsequent transcriptional activity. Rather than static independent influences, genetic variants may need only to be associated with initial aspects of nicotine exposure or other events, differentially initiating a cascade of subsequent effects. Interestingly, there is growing evidence that epigenetic markers can be passed down across generations and significantly affect offspring health 
[49]. Thus, modest individual variant findings may need to be explored in the context of epigenetics.

Taken together, like the ambiguities relating to specificity, it is both plausible and probable that the pathways from genome to syndrome are not a result of independent and additive effects, but interactive and recursive processes, reflecting much more complex dynamics than are typically examined. These relationships may in turn explain the observed inconsistencies in the literature.

\section{Understanding Genetic Influences via an Intermediate Phenotype Approach}

To a large extent, the preceding challenges reflect the common problem of a limited understanding of the proximal role that a genotype (or haplotype) has in either increasing or decreasing the probability of nicotine dependence. Although major technological advances have been made in terms of genotyping, the most common phenotypes continue to be clinical endpoints, such as nicotine dependence. Presence of a diagnosis or the level of dependence may be useful for describing a clinical syndrome and communication among clinicians, but may not be useful genetic phenotypes. This may be because a diagnosis or total index of symptoms is not highly informative about the disorder's underlying pathophysiology and motivational processes. Further, there are numerous pathways and symptom permutations that can lead to nicotine dependence and the syndrome may be considerably downstream from genome-level variation, making it an important clinical endpoint but an excessively diffuse phenotype.

One way to address this is an intermediate phenotype, or "endophenotype", approach, which attempts to characterize genetic influences by identifying intervening mechanistic processes (ie, the intermediate phenotypes or endophenotypes) that are responsible for a genetic variant's local influence on a disorder [50]. By focusing on more narrowly defined phenotypes that are associated with both genetic and clinical variation, this approach attempts to identify the proximal influences of risk-conferring and risk-preventing polymorphisms. Moreover, a focus on intermediate phenotypes has the potential to simultaneously identify the pathophysiologic and motivational mechanisms underlying the disorder, unlike the relatively opaque phenotype of diagnosis. Given the considerable phenotypic heterogeneity and limited evidence of large-magnitude single gene influences, this approach has considerable promise for clarifying the relationships between specific genes and nicotine dependence.

The objective of an intermediate phenotype approach is to map the risk pathway (positive or negative) from the genome to the clinical syndrome. This requires characterizing the role of genetic variation at multiple levels of analysis, from the level of transcription and the intra-/ intercellular functionality to emergent alterations of brain/body systems and, finally, proximal intermediate phenotypes that directly influence the clinical phenotype (Table 1). Broadly speaking, each of these levels reflects an important phenotype, but the level of proximal intermediate phenotypes is particularly important. These reflect processes that are sufficient (directly or indirectly) for explaining increases or decreases in motivation for nicotine. A recent study provides an excellent example and recapitulates the important levels of analysis. As illustrated in Table 1, Hutchison et al. [24••] found that a specific allele in the a 4 nAChR gene CHRNA4 was associated with significantly greater transcriptional activity in vitro, significantly greater smoking-induced reward in a human laboratory paradigm, and significantly greater nicotine dependence as a clinical phenotype. Thus, the study provides evidence that the polymorphism is functional at the level of transcription, that its functionality affects smoking reward (a motivationally relevant proximal intermediate phenotype), and this in turn affects the level of nicotine dependence. Applying this approach 
more broadly has the potential for fully understanding the role of an individual variant and in turn investigating its individual and interactive roles with other genetic and environmental factors.

Although its application to nicotine dependence is relatively new, a number of potentially useful intermediate phenotypes have emerged. These include tobacco-specific phenotypes, such as primary and secondary motives for smoking [26], relative value of tobacco [36], and smoking topography [13], and indirect phenotypes, such as cue-elicited craving [32] and impulsivity [43]. Shifting the phenotypic focus to motivationally relevant intermediate phenotypes that connect genetic variation and clinical variation has a number of potential advantages. A focus on intermediary genetic processes putatively increases the precision and validity of understanding how genetic risk is transmitted and may clarify interactive pathways that are typically overlooked. A mechanistic understanding of a polymorphism's role would contribute to understanding the boundary conditions of its influence (ie, for whom and under what conditions it is relevant). Finally, from an applied standpoint, intermediate phenotypes have the potential to be useful clinical targets for intervention or prevention.

\section{Conclusions}

There have been major advances in understanding the role of genetics in nicotine dependence. The condition is clearly heritable and has been found to be associated with a large number of individual genetic polymorphisms. However, inconsistent findings and typically small magnitude associations reveal the significant challenges posed to fully understanding genetic influences. Mapping these influences from individual loci to nicotine dependence, and in particular employing an intermediate phenotype approach, is a promising strategy for shedding light on a number of the extant challenges. In turn, a better understanding of the mechanisms of genetic risk and protection has great promise for improving prevention and treatment.

\section{Acknowledgments}

This review was partially supported by the following grants: National Institutes of Health (NIH) - K23 AA016936 (JM); NIH - R03 DA027481 (EMO, JM); NIH - P30 DA027827 (EMO, JM), NIH - R01 DA023134 (VSK). The authors are grateful for the research assistance of Anna Harrell, BS, and Shannen Malutinok, MSW, MPH.

\section{References}

1. World Health Organization. World Health Organization Statistics Report - 2008. Geneva, Switzerland: World Health Organization Press; 2008.

2. for Disease Control. Smoking-attributable mortality, years of potential life lost, and productivity losses-United States, 2000-2004. MMWR Morb Mortal Wkly Rep. 2008; 57:1226-1228. [PubMed: 19008791]

3. Henningfield JE, Miyasato K, Jasinski DR. Abuse liability and pharmacodynamic characteristics of intravenous and inhaled nicotine. J Pharmacol Exp Ther. 1985; 234:1-12. [PubMed: 4009494]

4••. Rose, RJ., et al. Genetics of smoking behavior. In: Kim, YK., editor. Handbook of Behavior Genetics. New York: Springer; 2009. p. 411-442.This chapter provides a full review of the quantitative genetic findings using twin methodologies

5. Li MD, et al. A meta-analysis of estimated genetic and environmental effects on smoking behavior in male and female adult twins. Addiction. 2003; 98:23-31. [PubMed: 12492752]

6. Morley KI, et al. Exploring the inter-relationship of smoking age-at-onset, cigarette consumption and smoking persistence: genes or environment? Psychol Med. 2007; 37:1357-1367. [PubMed: 17466111] 
7. Hamilton AS, et al. Gender differences in determinants of smoking initiation and persistence in California twins. Cancer Epidemiol Biomarkers Prev. 2006; 15:1189-1197. [PubMed: 16775180]

8. Mwenifumbo JC, Tyndale RF. Molecular genetics of nicotine metabolism. Handbook Exp Pharmacol. 2009; 192:235-259.

9. Swan GE, et al. Nicotine metabolism: the impact of CYP2A6 on estimates of additive genetic influence. Pharmacogenet Genomics. 2005; 15:115-125. [PubMed: 15861035]

10. Mwenifumbo JC, Sellers EM, Tyndale RF. Nicotine metabolism and CYP2A6 activity in a population of black African descent: impact of gender and light smoking. Drug Alcohol Depend. 2007; 89:24-33. [PubMed: 17161559]

11. Malaiyandi V, et al. CYP2A6 genotype, phenotype, and the use of nicotine metabolites as biomarkers during ad libitum smoking. Cancer Epidemiol Biomarkers Prev. 2006; 15:1812-1819. [PubMed: 17035386]

12. Schoedel KA, et al. Ethnic variation in CYP2A6 and association of genetically slow nicotine metabolism and smoking in adult Caucasians. Pharmacogenetics. 2004; 14:615-626. [PubMed: 15475735]

13. Strasser AA, et al. An association of CYP2A6 genotype and smoking topography. Nicotine Tob Res. 2007; 9:511-518. [PubMed: 17454707]

14. Munafò M, et al. The genetic basis for smoking behavior: a systematic review and meta-analysis. Nicotine Tob Res. 2004; 6:583-597. [PubMed: 15370155]

15. Lee AM, et al. CYP2B6 genotype alters abstinence rates in a bupropion smoking cessation trial. Biol Psychiatry. 2007; 62:635-641. [PubMed: 17223085]

16. Lee AM, et al. CYP2B6 genotype does not alter nicotine metabolism, plasma levels, or abstinence with nicotine replacement therapy. Cancer Epidemiol Biomarkers Prev. 2007; 16:1312-1314. [PubMed: 17548706]

17. Ring HZ, et al. Gene-gene interactions between CYP2B6 and CYP2A6 in nicotine metabolism. Pharmacogenet Genomics. 2007; 17:1007-1015. [PubMed: 18004205]

18. Yamanaka H, et al. Metabolic profile of nicotine in subjects whose CYP2A6 gene is deleted. Eur J Pharm Sci. 2004; 22:419-425. [PubMed: 15265511]

19. Chen G, et al. Glucuronidation of nicotine and cotinine by UGT2B10: loss of function by the UGT2B10 Codon 67 (Asp>Tyr) polymorphism. Cancer Res. 2007; 67:9024-9029. [PubMed: 17909004]

20. Benowitz NL. Neurobiology of nicotine addiction: implications for smoking cessation treatment. Am J Med. 2008; 121(4 Suppl 1):S3-S10. [PubMed: 18342164]

21. Berridge KC. The debate over dopamine's role in reward: the case for incentive salience. Psychopharmacology (Berl). 2007; 191:391-431. [PubMed: 17072591]

22. Tapper AR, et al. Nicotine activation of alpha $4 *$ receptors: sufficient for reward, tolerance, and sensitization. Science. 2004; 306:1029-1032. [PubMed: 15528443]

23. Brody AL, et al. Cigarette smoking saturates brain alpha 4 beta 2 nicotinic acetylcholine receptors. Arch Gen Psychiatry. 2006; 63:907-915. [PubMed: 16894067]

24••. Hutchison KE, et al. CHRNA4 and tobacco dependence: from gene regulation to treatment outcome. Arch Gen Psychiatry. 2007; 64:1078-1086. This article uses an intermediate phenotype approach to clarify the role of CHRNA4 polymorphisms in nicotine dependence on multiple levels of analysis. [PubMed: 17768273]

25. Bierut LJ, et al. Variants in nicotinic receptors and risk for nicotine dependence. Am J Psychiatry. 2008; 165:1163-1171. [PubMed: 18519524]

26. Baker TB, et al. Human neuronal acetylcholine receptor A5-A3-B4 haplotypes are associated with multiple nicotine dependence phenotypes. Nicotine Tob Res. 2009; 11:785-796. [PubMed: 19436041]

27••. Saccone NL, et al. Multiple independent loci at chromosome 15q25.1 affect smoking quantity: a meta-analysis and comparison with lung cancer and COPD. PLoS Genet. 2010 (in press). This meta-analysis reveals a highly significant association of cholinergic loci with smoking in a sample of over 38,000 individuals. 
28. Beuten J, et al. Significant association of catechol-O-methyltransferase (COMT) haplotypes with nicotine dependence in male and female smokers of two ethnic populations. Neuropsychopharmacology. 2006; 31:675-684. [PubMed: 16395295]

29. McKinney EF, et al. Association between polymorphisms in dopamine metabolic enzymes and tobacco consumption in smokers. Pharmacogenetics. 2000; 10:483-491. [PubMed: 10975602]

30. Huang W, et al. Significant association of ANKK1 and detection of a functional polymorphism with nicotine dependence in an African-American sample. Neuropsychopharmacology. 2009; 34:319-330. [PubMed: 18354387]

31. Huang W, et al. A functional polymorphism, rs6280, in DRD3 is significantly associated with nicotine dependence in European-American smokers. Am J Med Genet B Neuropsychiatr Genet. 2008; 147B:1109-1115. [PubMed: 18348205]

32. Hutchison KE, et al. The DRD4 VNTR polymorphism influences reactivity to smoking cues. J Abnorm Psychol. 2002; 111:134-143. [PubMed: 11866166]

33. Vandenbergh DJ, et al. Dopamine receptor genes (DRD2, DRD3 and DRD4) and gene-gene interactions associated with smoking-related behaviors. Addict Biol. 2007; 12:106-116. [PubMed: 17407504]

34. David SP, Munafò MR. Genetic variation in the dopamine pathway and smoking cessation. Pharmacogenomics. 2008; 9:1307-1321. [PubMed: 18781857]

35. Zhang L, Kendler KS, Chen X. The mu-opioid receptor gene and smoking initiation and nicotine dependence. Behav Brain Funct. 2006; 2:28. [PubMed: 16887046]

36. Ray R, et al. Association of OPRM1 A118G variant with the relative reinforcing value of nicotine. Psychopharmacology (Berl). 2006; 188:355-363. [PubMed: 16960700]

37. Munafò MR, et al. Association of the mu-opioid receptor gene with smoking cessation. Pharmacogenomics J. 2007; 7:353-361. [PubMed: 17224915]

38. Uhl GR, et al. Molecular genetics of successful smoking cessation: convergent genome-wide association study results. Arch Gen Psychiatry. 2008; 65:683-693. [PubMed: 18519826]

39. Hutchison KE, et al. Population stratification in the candidate gene study: fatal threat or red herring? Psychol Bull. 2004; 130:66-79. [PubMed: 14717650]

40. Grant BF, et al. Nicotine dependence and psychiatric disorders in the United States: results from the national epidemiologic survey on alcohol and related conditions. Arch Gen Psychiatry. 2004; 61:1107-1115. [PubMed: 15520358]

41. Kendler KS, et al. The structure of genetic and environmental risk factors for common psychiatric and substance use disorders in men and women. Arch Gen Psychiatry. 2003; 60:929-937. [PubMed: 12963675]

42. Kendler KS, Myers J, Prescott CA. Specificity of genetic and environmental risk factors for symptoms of cannabis, cocaine, alcohol, caffeine, and nicotine dependence. Arch Gen Psychiatry. 2007; 64:1313-1320. [PubMed: 17984400]

43. Eisenberg DT, et al. Examining impulsivity as an endophenotype using a behavioral approach: a DRD2 TaqI A and DRD4 48-bp VNTR association study. Behav Brain Funct. 2007; 3:2. [PubMed: 17214892]

44. Gelernter J, et al. Haplotype spanning TTC12 and ANKK1, flanked by the DRD2 and NCAM1 loci, is strongly associated to nicotine dependence in two distinct American populations. Hum Mol Genet. 2006; 15:3498-3507. [PubMed: 17085484]

45. Schmid B, et al. The interaction between the dopamine transporter gene and age at onset in relation to tobacco and alcohol use among 19-year-olds. Addict Biol. 2009; 14:489-499. [PubMed: 19740369]

46. Perkins KA, et al. Dopamine and opioid gene variants are associated with increased smoking reward and reinforcement owing to negative mood. Behav Pharmacol. 2008; 19:641-649. [PubMed: 18690118]

47. Renthal W, Nestler EJ. Epigenetic mechanisms in drug addiction. Trends Mol Med. 2008; 14:341350. [PubMed: 18635399]

48. Launay JM, et al. Smoking induces long-lasting effects through a monoamine-oxidase epigenetic regulation. PLoS One. 2009; 4:e7959. [PubMed: 19956754] 
49. Pembrey ME, et al. Sex-specific, male-line transgenerational responses in humans. Eur J Hum Genet. 2006; 14:159-166. [PubMed: 16391557]

50. Flint J, Munafò MR. The endophenotype concept in psychiatric genetics. Psychol Med. 2007; 37:163-180. [PubMed: 16978446] 


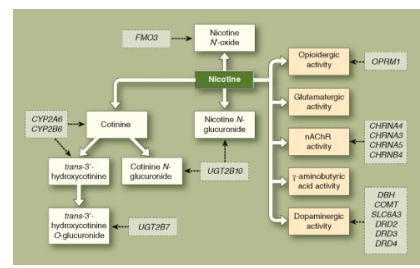

Figure 1. A model of the role of genetics in nicotine dependence via alterations to nicotine's pharmacokinetics and pharmacodynamics

Using continuous arrows, the pharmacokinetic pathways (blue) reflect the metabolic transformations of nicotine that determine its central and peripheral nervous system bioavailability and the pharmacodynamic pathways (green) reflect nicotine's molecular pharmacological effects on nicotinic acetylcholinergic receptors (nAChRs) and other neurotransmitter systems. Candidate genes (orange) and their points of putative influence are depicted using dashed arrows. Note that this is a simplified model of nicotine's pharmacokinetics and pharmacodynamics, and the candidate genes presented are illustrative examples, not an exhaustive list. 


\section{Table 1}

Mapping genetic risk from genome to syndrome using an intermediate phenotype approach

\begin{tabular}{lll}
\hline Level of analysis & Observed variation & Example: Hutchison et al. [24••. $]^{\boldsymbol{a}, \boldsymbol{b}}$ \\
\hline Genome & Polymorphism & $\mathrm{G} \rightarrow$ A SNP (rs6122429) \\
Intra-/Intercellular milieu & Transcription/biochemical & $\mathrm{GG}=\uparrow$ binding \\
Physiologic system (body/brain) $b$ & System/network & $\mathrm{GG}=\uparrow$ a4* NAChR/DA activity (putative) \\
Proximal intermediate phenotype & Motivational & $\mathrm{GG}=\uparrow$ smoking reward \\
Clinical & Presence/severity & $\mathrm{GG}=\uparrow$ nicotine dependence/smoking \\
\hline
\end{tabular}

${ }^{a}$ A guanine (G) to adenine (A) substitution at position 4490 in the CHRNA4 gene is a single nucleotide polymorphism (SNP) for which homozygous $\mathrm{G}$ genotype was found to be associated with increased binding, subjective smoking reward, and nicotine dependence.

${ }^{b}$ A level of analysis not examined in the study was the physiologic system, where the observed transcriptional variation putatively alters cholinergic and/or dopaminergic activity. 